\title{
NARRATIVAS PERIODÍSTICAS: LA AGENDA SETTING Y LAS CONSECUENCIAS DE LAS NOTICIAS SOBRE LA FIGURA DEL PRESO
}

\author{
NARRATIVES IN FOCUS: THE SETTING AGENDA AND THE PUBLIC \\ EFFECTS OF THE NEWS ON THE PRISONER'S FIGURE
}

\author{
NARRATIVAS JORNALÍSTICAS: A AGENDA SETTING E AS \\ CONSEQUÊNCIAS DAS NOTÍCIAS SOBRE A FIGURA DO PRESO
}

\author{
Francisco Gilson Rebouças Porto Junior \\ Universidad Federal de Tocantins, Brasil \\ http://orcid.org/0000-0002-5335-6428 \\ gilsonportouft@gmail.com
}

\author{
Giliarde Albuquerque Cavalcante Virgulino Ribeiro Nascimento \\ Universidad Federal de Tocantins, Brasil \\ https://orcid.org/0000-0001-8146-6811 \\ gi.albuquerquecavalcante@gmail.com
}

Recibido: 10 /06 /2020 Revisado: 18/06/2020 Aceptado: $30 / 06 / 2020$ Publicado:01/11 /2020

Resumen: Este artículo se centra en la construcción de narrativas sobre los efectos públicos de la figura del preso difundido o temático en la construcción de narrativas periodísticas, realizadas por Jornal do Tocantins. Con este fin, se procedió a la recopilación de datos para la solidez de las discusiones a través de una encuesta en línea, que trajo a colación temas relacionados con las noticias del vehículo de estudio periodístico, las percepciones de la sociedad y las posibles consecuencias reflejas para el preso, permitiendo materializar los objetivos que se basan en lecturas críticas y reflexivas de efectos negativos ciertos de las narrativas periodísticas para las personas privadas de libertad en Brasil. Por lo tanto, es una investigación cuantitativa basada en datos numéricos y en su tratamiento por literatura, principalmente, de Shaw que convergen para actualizar la discusión sobre el lugar de la pretensión (ambiente carcelario). En conclusión, se confirma que las personas encuestadas pueden comprender lo que es libertad de prensa; su ejercicio por el Jornal do Tocantins; los derechos personales (imagen, privacidad, intimidad) del preso; y las consecuencias de ciertas narrativas que no prestan atención al valor noticia adoptado, siendo un riesgo a la credibilidad de las actividades periodísticas.

Palabras claves: Ajuste de agenda; Narrativas; Comunicación; Periodismo.

Abstract: This article focuses on the construction of narratives about the public effects of the figure of the prisoner disseminated or themed in the construction of journalistic narratives, in casu, made by Jornal do Tocantins. To this end, we proceeded with the collection of data for the robustness of the discussions, which was carried out through an online survey that took place with the application of a questionnaire directed on a digital platform, which brought up issues involving the great researched topic (Agenda Setting and trapped population). Therefore, it is clear that this is a qualitative research based on specific literature and / or in-depth studies by Maxwell E. McCombs and Donald L. Shaw and others that converge to update the discussion for the locus of pretension (Tocantins prison environment). The conclusions reached with force in empirical data raised even though they deal with sufficiency of the subject or central theme, 


\section{Hachetetepé. Revista científica de Educación y Comunicación}

$\mathrm{n}^{\circ} 21,15-31,2020$

making it possible to understand and comprehend in a broader way the effects of Agenda Setting theorizations in a prison environment and in the formation of public opinion.

Keywords: Agenda Setting; Narratives; Communication; Journalism.

Resumo: O presente artigo tem como foco a construção das narrativas sobre os efeitos públicos da figura do preso disseminada ou tematizada em construções de narrativas jornalísticas, in casu, feitas pelo Jornal do Tocantins. Para tanto, procedeu-se com o levantamento de dados para robustez das discussões por meio de pesquisa online, que trouxe à baila questões envolvendo as notícias do veículo jornalístico de estudo, as percepções da sociedade e as possíveis consequências reflexas ao preso, permitindo materializar os objetivos que se baseiam em certos efeitos negativos das narrativas jornalísticas para as pessoas privadas de liberdade no Brasil. Portanto, se trata de pesquisa quantitativa que se apoia em dados numéricos e no em tratamento destes por literatura específica e/ou de aprofundamento dos estudos de McCombs e Shaw que convergem para a atualização da discussão para o locus de pretensão (ambiente carcerário tocantinense). Em conclusão, se confirma que as pessoas pesquisadas são capazes de compreender o que se tem por liberdade de imprensa, seu exercício pelo Jornal do Tocantins, os direitos personalíssimos (imagem, privacidade, intimidade) do preso e consequências de certas narrativas que não prestam a devida atenção na eleição de valores-notícia, sendo um risco à credibilidade das atividades jornalísticas.

Palavras-chave: Agenda Setting; Narrativas; Comunicação; Jornalismo.

Cómo citar este artículo: Porto Junior, F.G.R. y Nascimento, G.A.C.V.R (2020). Narrativas periodísticas: la Agenda Setting y las consecuencias de las noticias sobre la figura del preso. Hachetetepé. Revista científica en Educación y Comunicación, (21), 15-31. https://doi.org/10.25267/Hachetetepe.2020.i21.3

\section{INTRODUCCIÓN}

La evolución de los estudios sobre Agenda Setting o Teoría de la Planificación de Agenda, memorable desde 1970, originalmente es un producto de contribuciones de McCombs y Shaw, resaltando en la actualidad el enfrentamiento de nuevas perspectivas y conceptos que se remodelan con el avance de nuevas construcciones culturales, sobrepujando sobrepasar ámbitos o ambientes (comunicativos, políticos, sociales, etc.). Frente a eso, es preciso atenerse a nuevas interacciones sectorizadas que avancen en los estudios sobre Agenda Setting (Formiga, 2006).

Considerando las nuevas posibilidades discursivas de las teorías de McCombs y Shaw, cuyo estudio de angulación aquí introducirse en un delicado escenario de control social, la cárcel, ambiente que trae implicaciones de variadas órdenes, por ejemplo, la libertad de prensa, la ética periodística, los derechos de la población presa, que no se contemplan en edicto de prisión o condenación... estos ejemplos de vectores reclamantes de profundización investigativa dirigida $\mathrm{y}$, sin duda, todos con marcas claras de los procesos comunicativos que revelan el modus operandi que la teoría de la planificación de agenda viene mostrando y (re)construyéndose (Porto Junior y Moraes, 2017; Nunes et al., 2017; Reis, 2017; Moio y Vieira, 2017; Melo, 2017; Rovai, 2017; Martinez, 2016; Alonso, 2016; Almeida, 2016).

Las reinvenciones o (re)construcciones teóricas del Agenda Setting van explotando nuevos espacios de producción y distribución de la información, condición íntima al proprio sistema informativo de consumo. Desde este punto en adelante, los códigos de la 


\section{Hachetetepé. Revista científica de Educación y Comunicación}

$\mathrm{n}^{\circ} 21,15-31,2020$

lógica comunicacional avanzan hacia espacios virtuales, no dictándose solo por el consumo en masa y formación de la esfera pública, sino, además, por el locus de construcción y diseminación de la información (Lemo, 2009).

Así, se busca conocer en este trabajo el locus de construcción de la información y de los elementos o particularidades que se activarse avivan en el referido ámbito, todo para analizar los niveles y elementos de la teoría, en contrapunto con el ambiente carcelario.

Así, se elige el ambiente carcelario tocantinense como locus de la construcción informativa, construcción que, en el presente estudio, se da por el Jornal de Tocantins, vehículo totalmente online y con expresiva actividad periodística y de formación de opinión en el Estado de Tocantins.

Instalándose en el locus de la investigación y del medio de producción y diseminación de la información, sin apego a producciones específicas, pero sí a la confirmación o refutación de interacciones que vengan a contrastar los niveles y elementos del Agenda Setting, o sea, los efectos públicos sobre la figura del preso divulgada en construcciones de narrativas periodísticas, in casu, hechas por el Jornal de Tocantins.

Para eso, se procedió al análisis levantamiento de datos para para dotar de solidez a las discusiones, lo que se operó por medio de una investigación online que se dio con la aplicación de un cuestionario dirigido en la plataforma digital, Google Forms, que trajo a escena cuestiones que envuelven el gran tema investigado (Agenda Setting y población presa).

Por tanto, se trata de una investigación cuantitativa que se apoya en literatura específica y de profundización de los estudios de McCombs y Shaw que convergen para la actualización de la discusión para el locus de pretensión (ambiente carcelario tocantinense).

\section{METODOLOGÍA}

La investigación realizada fue una revisión bibliográfica y cuantitativa. La investigación se basó en datos estadísticos recopilados en una plataforma virtual sobre temas relacionados con la libertad de prensa ejercida por Jornal do Tocantins (periódico en línea) y la población encarcelada en Tocantins, Brasil, informada por ese vehículo. Cuando se recopilaron los datos, se trataron críticamente mediante un proceso evaluativo de revisión bibliográfica de un estudio realizado por McCombs y Donald L. Shaw, que se muestran en la Web, exponentes de la Teoría de la configuración de la agenda en investigaciones de todo el mundo (Mccombs, 2004; Gil, 1991).

Para la recolección de datos se eligió el cuestionario. Este resultó ser suficiente para proporcionar información adecuada y necesaria para alcanzar el objetivo principal, a saber, conocer el papel de Jornal do Tocantins en la construcción de narrativas sobre prisioneros y las consecuencias de los discursos sobre la opinión de las personas encuestadas (Gerhardt y Silveira, 2009).

El cuestionario se aplicó en la plataforma virtual y gratuita, Google Forms. El cuestionario se tituló "Libertad de prensa versus derechos de la población carcelaria" y contenía 07 preguntas numeradas del 01 al 07, con alternativas objetivas de "sí" o "no" para todos los ítems, con la excepción de las preguntas 01 y 02 , teniendo 03 alternativas cada una, ambas variando solo en la tercera alternativa: "no sé" para la pregunta 02 ; y "tal vez" para la pregunta 03 . 


\section{Hachetetepé. Revista científica de Educación y Comunicación}

$\mathrm{n}^{\circ} 21,15-31,2020$

A continuación, las preguntas 01 a 07 se presentan en el cuestionario aplicado de acuerdo con las consideraciones y aclaraciones anteriores.

Pregunta 01. ¿Considera que las noticias de Jornal do Tocantins son confiables? Pregunta 02. ¿Te consideras influenciado por los medios? Pregunta 03. ¿Has leído noticias de Jornal do Tocantins sobre crisis en el sistema penitenciario? Pregunta 04. ¿El prisionero también disfruta de derechos muy personales (imagen, privacidad, intimidad)? Pregunta 05. ¿Sabes qué es la libertad de prensa? Pregunta 06. ¿Considera que los medios de comunicación representan a la población carcelaria como abusiva? Pregunta 07. ¿Considera que la libertad de prensa es un derecho de los periódicos brasileños, y que estos medios de comunicación, en nombre de la libertad de prensa, pueden tratar sus narrativas sobre la imagen, la privacidad y la intimidad del prisionero?

El cuestionario con las preguntas presentadas se puso a disposición en línea, para participación voluntaria y gratuita, enviando invitaciones a investigadores de grupos de profesionales de WhatsApp de áreas relacionadas con el tema de investigación.

El público objetivo elegido para la aplicación del cuestionario fue formado por profesionales de la Comunicación de Tocantins; el Sistema Penitenciario de Tocantins; el Colegio de Abogados de Brasil; la Oficina del Defensor Público de Tocantins; y la Oficina del Fiscal Federal en Tocantins.

Optamos por la aplicación a grupos específicos de profesiones relacionados con el tema de investigación (Periodismo, Derecho, Ejecución Penal) para comprender más de cerca cómo estas personas, que son importantes en el proceso de dirigir las opiniones generales del cuerpo social, se comportan frente al problema.

Los resultados obtenidos del posicionamiento de profesionales de estos sectores profesionales sirven como un parámetro para leer el comportamiento general y para la interpretación de la posible potencialización de las consecuencias sociales sobre la figura de la persona arrestada, que surge de comportamientos profesionales desatendidos, no ajustados y que confirman programación de comportamientos en el tejido social (Lemo, 2009; y Demo, 1994).

La contribución a la investigación, a través de un cuestionario en línea y una participación voluntaria y gratuita, tuvo lugar durante el período del 15/03/2020 al $15 / 04 / 2020$ y requirió la disponibilidad de un correo electrónico personal de cada informante, como condición para comenzar el cuestionario, con el único y exclusivo propósito de registrar la participación, además de para completar el cuestionario y enviar los datos y las respuestas a todas las preguntas del 01 al 07.

Al final del período de recopilación de datos, se registraron 57 cuestionarios, que se iniciaron, completaron y enviaron los resultados en Formularios de Google.

Tal como se presentó, se decidió utilizar un cuestionario con preguntas cerradas, no permitiendo que cada persona investigada se posicionara fuera de las alternativas de los ítems de respuesta disponibles (sí, no, no lo sé y tal vez), para contribuir a la recopilación de datos estadísticos capaces de proporcionar un análisis de resultados que pudieran mostrar posibles fenómenos individuales, grupales y organizacionales, sociales y políticos (Lemo, 2009; Demo, 1994).

Todos los ítems del cuestionario se organizaron con un nivel creciente de profundización teórica de la investigación, con el objetivo de facilitar al investigador el proceso de organización y comprensión lógica de las diversas respuestas proporcionadas y tratadas (Lemo, 2009; Demo, 1994). 


\section{LA AGENDA SETTING: NIVELES, ELEMENTOS Y LOCUS OPERANDI}

Se puede apuntar al siglo XX, en la historia de la Comunicación, como el periodo de comienzo de estudios que respaldaron e influyeron en las teorías de la comunicación conocidas en la actualidad, siendo la radio y la televisión las primeras tecnologías del área a ser explotadas de forma científica. Si hacemos búsquedas de los primeros rastros de investigaciones de comunicación, se observará que los Estados Unidos figuran como principal campo de nacimiento (Martino, 2010).

Los estudios observados en la red mundial de ordenadores se centran, inicialmente, en la Universidad de Columbia. Así, se reunieron contribuciones de diversos investigadores de áreas diversas, destacándose investigadores de sociología, psicología, ingeniería, comunicación, etc., con considerables aproximaciones de factores políticos y económicos como centros de aproximación del objeto comunicacional, (parcialidad utilitarista), esfuerzos favorecedores de la investigación acerca de los procesos de comunicación (Martino, 2010; Araújo, 2008; França, 2008).

No obstante, a pesar de que los factores políticos y económicos bajo una influencia, utilitarista le revisten tónicamente, el campo de la comunicación comenzó a revelar unidad, es decir, características comunes en estudios entre 1920 y 1960 que apuntaban hacia una organización autónoma del conocimiento.

Son considerados factores o elementos de unidad a la Comunicación, orientación empírica, orientación pragmática, objeto de estudio propio, fundamentación bajo el modelo comunicativo de Harold D. Lasswell (Araújo, 2008).

Con la introducción hecha, se llega al Agenda Setting, con la evidencia de que sus trabajos iniciales o estudios se dieron en tierras norteamericanas, pues, como ya se ha apuntado, los Estados Unidos de América fueron considerados la cuna de la Comunicación.

Hecho esto, es preciso tratar sobre las teorías de la Mass Comunication Research locus en que se localiza, por consecuencia, la Teoría de la Planificación de Agenda. Dicha clase de teorías trata sobre los efectos de los medios de comunicación sobre sujetos sociales o, simplemente, sujetos, individuos, sociedad (Wolf, 2002).

En 1968, McCombs y Shaw, de la Universidad de Carolina del Norte - EE.UU., unieron fuerzas para iniciar investigaciones sobre la influencia de los mass media sobre la opinión pública, de la influencia de los medios de comunicación en ciertos intereses sociales reflejos en debates públicos. Pasado un tiempo, fruto de los esfuerzos fue la publicación del artículo titulado The Agenda-Setting Function of Mass Media en la Public Opinion Quarterly. Sin embargo, los estudios demostraron limitaciones que, al fin y al cabo, no comprometieron los resultados sino que exigirían profundizaciones posteriores en los objetos del levantamiento empírico (Mccomboms, 2004; Formiga, 2006).

Las profundizaciones sobre la Agenda Setting están ocurriendo en todo el mundo desde los estudios iniciales de 1970, pues las referidas profundizaciones son la promesa de rediscusión de elementos teóricos que requieren aplicación o test en contextos y escenarios distintos de la sociedad como un todo, siendo al final lo que se pretende aquí, el ambiente carcelario fructífero en términos de contribuciones y confirmaciones de la teoría bajo examen.

Martino (2010) atribuye a las pesquisas de planificación un marco de orientación de los efectos de la comunicación en masa, pues, como dice, el objeto de estudio centralizador no fue la persuasión, sino la relación causa y tiempo del efecto de la planificación. 
A menudo, la función de la planificación fue puesta como la facultad que los espacios de comunicación o medios de comunicación masivos enfatizan pautas o temas al punto de ser acotados como temas de discusión de la sociedad. No es posible decir si, actualmente, los mass media son completamente responsables de la definición de comportamientos pero, al menos, es evidente que son capaces de influir en comportamientos pre-objetivados (Mccombs y Shaw, 1972).

Se puede afirmar que los niveles de agenda mediática orbitan en la Agenda Setting, la agenda pública y la agenda de políticas públicas, además de influir en la relación de elementos como, frame temporal, time-lag, centralidad, valor individual, etcétera. Alguna que otra vez, se consideran como fases: premisa básica y de centro (facultad de los medios de comunicación de planificar/definir los asuntos y su relevancia en el espacio público); los efectos psicológicos y/o sociológicos de la planificación (necesidad social de orientación); planificación de atributos (temas públicos reclamados); los factores de modelación de la agenda mediática; los efectos asociativos de temas públicos y las imágenes de construcción personal o la incorporación de los objetos y de la definición de relevancia del tema en simultaneidad (Mccombs, 2004; Lippman, 2008; Luo, 2011; Barros Filho, 1995).

El debate hasta ahora ha hecho posible algunas ponderaciones al respecto de los niveles, elementos y locus operandi de la Teoría de la Planificación, teniéndose, además, abordado en la medida de lo que importa a la proposición investigativa y teórica de estudios sobre la definida teoría.

De aquí en adelante, el citado conjunto de formación de la Agenda Setting será mejor explorado con la presentación de datos empíricos de colecta en campi virtual, los cuales relacionan el ambiente carcelario, el Jornal de Tocantins y los efectos de noticias, lato sensu, sobre la opinión pública de la figura del preso.

\section{NOTAS SINÓPTICAS AL AMBIENTE CARCELARIO TOCANTINENSE}

En líneas generales, conviene trabajar el nacimiento de la idea de prisión, cárcel, penitencia, ergástulo,... una misma idea con varios términos que hablan sobre la congruente situación: el aprisionamiento del ser humano.

El aprisionamiento del ser humano, que surge con el desvalor de la conducta humana por parte del Estado, en la actualidad y, que, como respuesta, designa la pérdida o restricción del ius libertatis, requiere una mirada de fuera de lo que le otorgó primacía, el capitalismo, la negación a la pérdida de mano de obra frente a penas que resultaban en muerte del individuo (Bitencourt, 2016; Foucault, 1999).

Las prisiones se muestran como locales para el cumplimiento o ejecución de la pena que priva a un individuo de la libertad humana. Hay que recordar que esa consecuencia sobre el individuo social surge inspirada en modelos de la institución de la iglesia, cuando en tiempos no precisos, los fieles sufrían penas de penitencia, siendo aislados en celdas. En el sistema capitalista, se acordó para vigilar sobre el individuo de manera utilitarista con la noción de desperdicio de mano de obra, fuerza de trabajo que, al fin y al cabo, fuesen provechosas a los proyectos de desarrollo capitalista (Oliveira, 1977; Ferreira, 2000; Bitencourt, 2016; Miotto, 1970). 


\section{Figura 1}

Unidades Prisionais no Tocantis

\section{ESTABELECIMENTOS PRISIONAIS TOCANTINS}

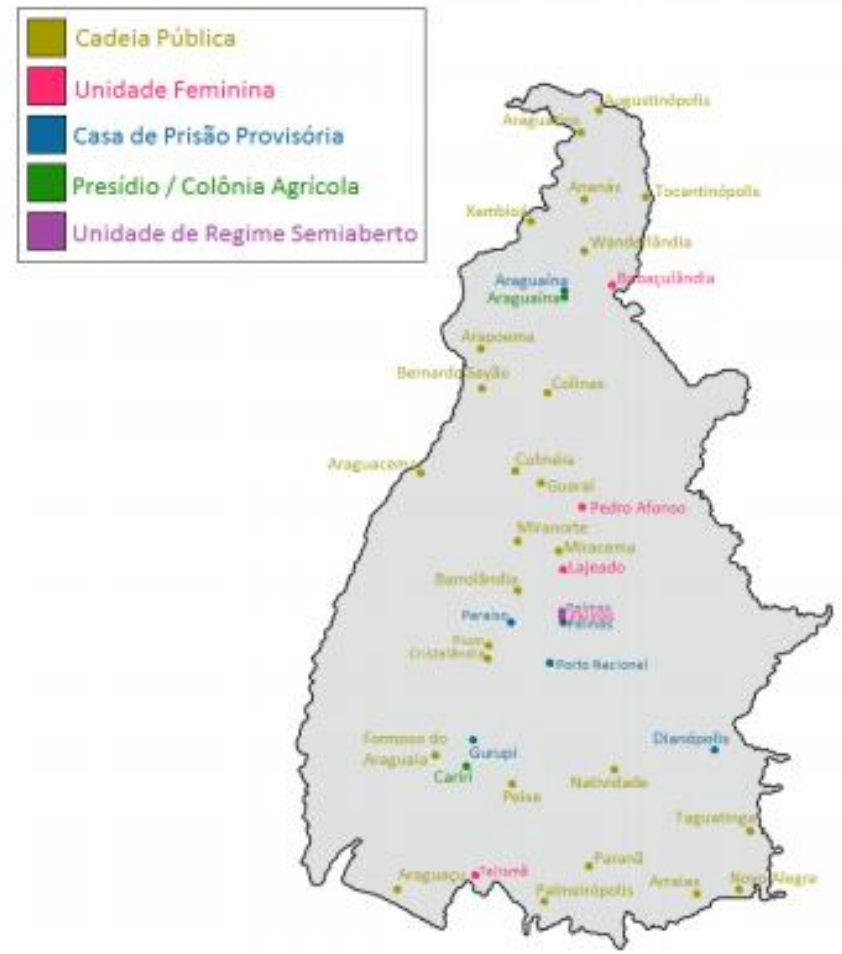

Fuente: Plano de Adecuación de Recursos Humanos y de Unidades del Sistema de Prisiones de Tocantins (SECIJU, 2017).

Con lo pincelado, volvamos al escenario regionalizado o de estado de la federación, en concreto, de la cárcel en Tocantins. Las prisiones tocantinenses están bajo la responsabilidad de la Secretaría de Estado de la Ciudadanía y Justicia

(...) la Secretaría de Ciudadanía y Justicia (...) recibió la atribución de gerenciar el sistema penitenciario, competiéndole "elaborar, coordinar y administrar la política de prisiones por medio de la custodia de los individuos privados de libertad, promoviendo condiciones efectivas para la reintegración social, tal como previsto en la Ley 2.461, de 7 de julio de 2011(SECIJU, 2017, p. 3).

Las unidades de prisión en Tocantins, de acuerdo con el Plan Director del Sistema Penitenciario de Tocantins (PDSPTO, 2008), estaban en número de 21 unidades, incrementándose para 44 (cuarenta y cuatro) unidades en el año 2017. En el mapa infra ofrece mayor detalle sobre la naturaleza de las unidades.

Como demostrado, de las 44 unidades de prisión, 33 (treinta y tres) son prisiones públicas masculinas, 06 (seis) (femeninas), 02 (dos) penitenciarías y 03 (03) de monitoramiento electrónico.

En lo referente al número de personas mantenidas en las unidades de presidio, la Secretaría de Ciudadanía y Justicia de Tocantins, por medio del Plan de Adecuación de 
Recursos Humanos y de Unidades del Sistema de Presidíos de Tocantins, de 2017, tratan del tema.

El excesivo número de pequeños establecimientos de prisión dificulta sobremanera la adopción de medidas de cuño de resocialización tales como: escuelas, talleres de trabajo y otras actividades afines, una vez que esas unidades no poseen estructuras adecuadas para este tipo de política. De esta forma, ni la misma la separación por clasificación penal en conformidad con la LEP puede ser realizada, perjudicando el combate a la reincidencia delictiva (SECIJU, 2017, pp. 13- 15).

De acuerdo con el Plan, Tocantins contaba con 3.844 (tres mil, ochocientos y cuarenta y cuatro) personas presas en 2017.

Por todo esto que se trilló, se llega a la conclusión de esa partición que buscó situar el ambiente carcelario en estructura, naturaleza y números (personas presas).

Ahora se entiende posible entrelazar discursivamente la Teoría de la Planificación y el ambiente carcelario para entender percepciones de ciertas personas encuestadas a las noticias que tienen a las persona presas como figuras de noticia.

\section{PRESENTACIÓN Y DISCUSIÓN DE LOS DATOS EMPÍRICOS DE LA INVESTIGACIÓN}

Se considera que la recepción de un hecho o acontecimiento al punto de tornarse relevante en algún grado en nuestro "mapa cognitivo" se da a partir del instante en que contactamos de alguna manera, presencial o no. Con eso, numerosos otros eventos ocurren en varios otros espacios, países, continentes, no recibiendo demás hechos y eventos de igual importancia o ni si quiera la ganan por parte de cada uno de nosotros (Mccombs, 2004).

Son los medios de comunicación, por así decirlo, el enlace de aproximación de muchos de los hechos del mundo para diferentes individuos, a pesar de que estos presentan limitada capacidad de intelección de todos los hechos existentes. La comunicación es responsable, de esta manera, de permitir que estos formen part del día a día de las personas. De esta forma, se aprovechan los medios de comunicación en masa del espacio intangible de la mente humana uniendo personas y hechos que ocurren en cualquier lugar del mundo. Así, verbi gratia, una persona del país $\mathrm{X}$ puede discutir asunto del país Y, todo sin precisar estar en ninguno de los dos países (Lippman, 2008).

Por lo tanto, es evidente la imposibilidad de atenernos a todos los hechos del mundo, razón por la cual el análisis de la Agenda Setting requiere una aproximación con eventos en un número reducido, siendo la propuesta de ese estudio aquellos datos que han de contribuir a la discusión.

Todos los datos se dan con la compilación de los resultados de la pesquisa "libertad de prensa versus derechos de la población presa" (base de la pesquisa en Google Forms), que se basó en la aplicación de un cuestionario, el cual abordó 07 cuestiones que reclamaron del público pesquisado (servidores del Sistema de Prisiones y Penitenciario de Tocantins, profesionales del derecho - abogado, defensor público, procurador federal de justicia -y profesionales de la comunicación - periodistas) el posicionamiento en relación a temas considerados orbitales a la teorización de la Agenda Setting, todos oportunamente presentados y debatidos a lo largo de esta partición.

En total se obtuvieron 57 resultados que propician un tratamiento científico sólido. Así, vale decir que las respuestas al formulario exigieran el uso obligatorio de una 
dirección de e-mail valido y/o activo para registro oficial, lo que contribuye confiabilidad a los resultados.

Además, todas las respuestas iniciadas exigían posicionamiento en todos los ítems, los cuales poseían como alternativa SÍ o NO como posicionamientos admitidos y definitivos, a excepción de los ítems 01 y 02 de un total de 07 que admitían las alternativas "desconozco y tal vez", respectivamente, ante particularidades confluentes al tratamiento final de los datos.

En la presentación de las cuestiones abordadas en el formulario y digresiones científicas sobre los objetos investigados.

\section{Ítem 01 - “CONSIDERA LAS NOTICIAS DEL JORNAL DE TOCANTINS} CONFIABLES? “.

De las 57 (cincuenta y siete) respuestas registradas, 47,4\% se respondieron con SÍ, $36,8 \%$ registradas con NO y $15,8 \%$ registradas con DESCONOZCO.

\section{Figura 2}

Resultado del ítem 01

1. CONSIDERA AS NOTICIAS DO JORNAL DO TOCANTINS CONFIÁVEIS?

57 respostas

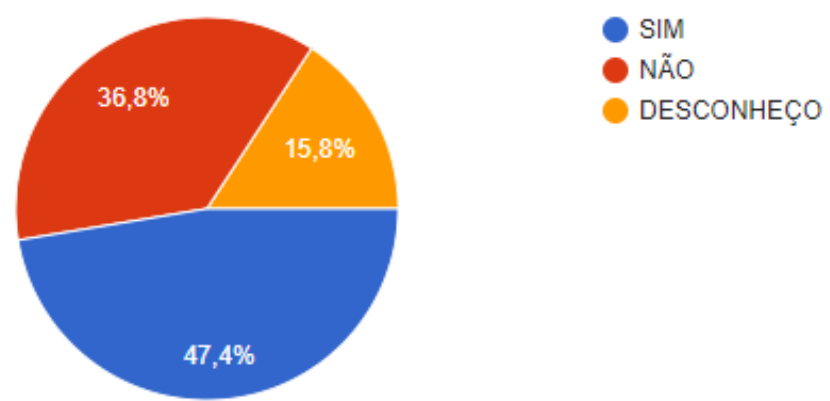

Los resultados expresan considerable inseguridad o credibilidad en relación con la actuación periodística del referido periódico, viendo que 47,4 \% afirman no ver como confiables las noticias del medio de comunicación en discusión, lo que coincide con la posible falta del medio en robustecer el valor-noticia y/o planificación que alinee el interés público sobre la temática. 


\section{Ítem 02 - “¿USTED SE CONSIDERA INFLUENCIADO (A) POR LOS MEDIOS DE COMUNICACIÓN?"}

De las 57 (cincuenta y siete) respuestas registradas, $71,9 \%$ fueran registradas con NO, 22,8 \% registradas con TAL VEZ y 5,3\% registradas con SÍ.

\section{Figura 3}

Resultado del ítem 2

2. VOCÊ SE CONSIDERA INFLUENCIADO (A) PELA MIDIA?

57 respostas

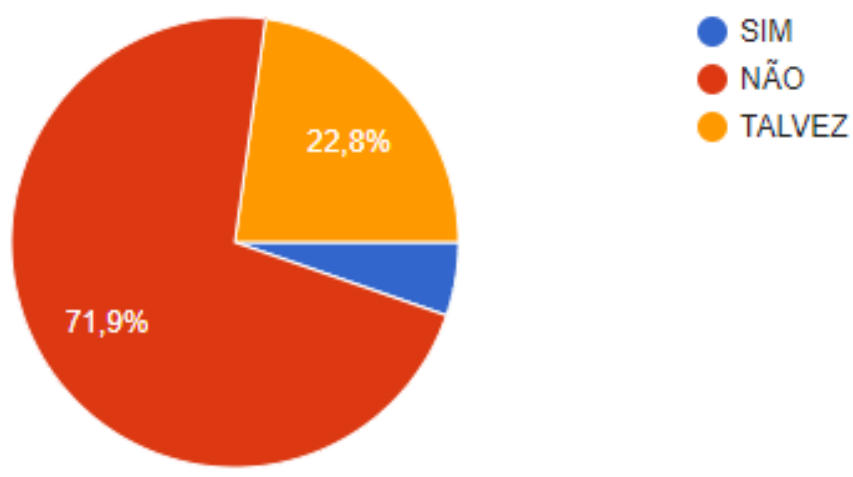

Cuando los investigados son preguntados sobre la influencia que consideran sufrir de los mass media, el 71,9\% de los sujetos investigados se consideran no influenciables.

La afirmación mayoritaria no se sostiene en los postulados de la Teoría de la Planificación, pues, conforme a los estudios de Lippman (2008), los medios de comunicación tienen un importante y papel central en la distribución de valores o estímulos para la creación de imágenes de la realidad. El propio investigador considera que los mass media no los únicos influyentes, aunque son los más destacados.

McCombs (2004) afirma que la importancia de la planificación se da enfatizando temas o noticias, que acabarán tornándose relevantes con el paso del tiempo conforme al público receptor. 


\section{Ítem 03 - “¿USTED YA HA LEÍDO NOTICIAS DEL JORNAL DE TOCANTINS SOBRE CRISIS EN EL SISTEMA DE PRISIONES?}

De las 57 (cincuenta y siete) respuestas registradas, 64,9\% fueron registradas con SÍ y $35,1 \%$ registradas con NO.

\section{Figura 4}

Resultado del ítem 03

\section{VOCÊ JÁ LEU NOTICIAS DO JORNAL DO TOCANTINS SOBRE CRISES NO SISTEMA PRISIONAL? \\ 57 respostas}

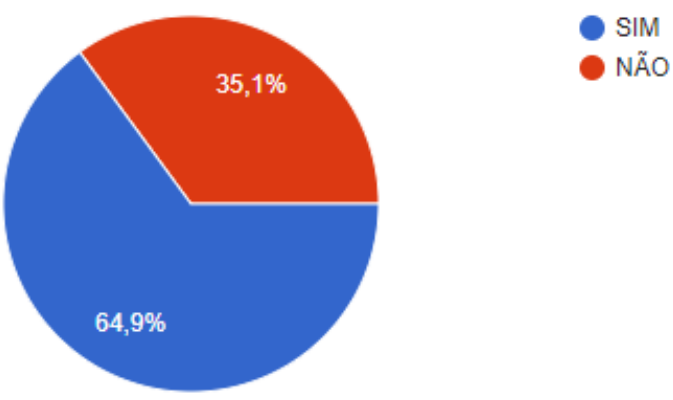

Para la situación, 64,9 \% (ahora más del $50 \%$ de los resultados) del público evaluado dice ya haber leído o conocido alguna noticia del periódico relacionado con crisis en el sistema de presidios. Este dato evidencia que las noticias de crisis en el sistema de prisiones pueden mapear mentes, viendo, que el número mayoritario dice recordarlo, lo que confirma el efecto planificación.

Al confirmar la planificación de noticias de crisis en las prisiones por parte del vehículo bajo estudio, el dato cobra mayor preocupación con la manera de la construcción de las narrativas que envuelven el tema central, ante la delicadeza del ambiente carcelario y límites tal vez no conocidos de la actuación periodística al tratarse, especialmente, de los derechos de la persona presa.

De acuerdo con el modelo de McCombs (2004), se puede encuadrar la preocupación del Jornal al tema discutido en la primera fase de la planificación, es decir, la fase en que se ejerce la capacidad, por el medio de comunicación, de planificar el asunto que considera relevante, formando así la agenda mediática que resultará en la consideración de lo que es importante en la agenda pública. Con todo, se incide en que la planificación debe dirigirse por medio de los valores de la noticia y de la actuación ética, cuando se respetan los límites de la actuación. 


\section{Ítem 04 - “¿LA PERSONA PRESA TAMBIÉN GOZA DE LOS DERECHOS PERSONALISIMOS (IMAGEN, PRIVACIDAD, INTIMIDAD)?”}

De las 57 (cincuenta y siete) respuestas registradas, 71,9\% fueron registradas con SÍ y $28,1 \%$ registradas con NO.

\section{Figura 5}

Resultado del ítem 04

\section{A PESSOA PRESA TAMBÉM GOZA DOS DIREITOS PERSONALISSIMOS (IMAGEM, PRIVACIDADE, INTIMIDADE)? \\ 57 respostas}

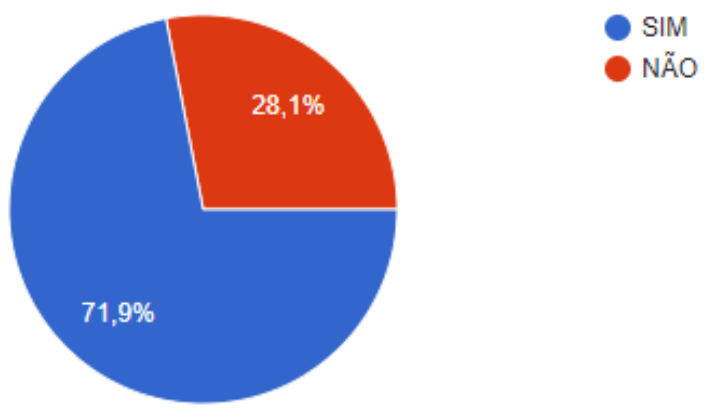

Ya que se discute el ambiente carcelario, la relevancia debe darse sobre los individuos allí mantenidos, se habla de la figura del preso que sí posee derechos que deben ser respetados, citando, derecho de imagen, de privacidad y de intimidad.

Como mínimo, el Estado no garantiza la estructura interna capaz de ofrecer al custodiado acceso a condiciones básica de supervivencia, aire, iluminación e instalaciones sanitarias (Mirabete, 1996). Entonces, es difícil imaginar que los derechos personales son respetados, frente a la ineficiencia de la garantía de lo mínimo existencial.

Citados los derechos que precisan ser considerados en la formación narrativa periodística, bajo posibilidad de incursión en violación de límites que la ética periodística ya advierte, pues, como ya observamos, el 71,9 \% del público afirma conocer tales derechos. 


\section{Ítem 05 - “¿USTED SABE LO QUE ES LIBERTAD DE PRENSA?”}

De las 57 (cincuenta y siete) respuestas registradas, $93 \%$ fueron registradas con SÍ y $7 \%$ registradas con NO.

\section{Figura 6}

Resultado del ítem 05

\section{VOCÊ SABE O QUE É LIBERDADE DE IMPRENSA? \\ 57 respostas}

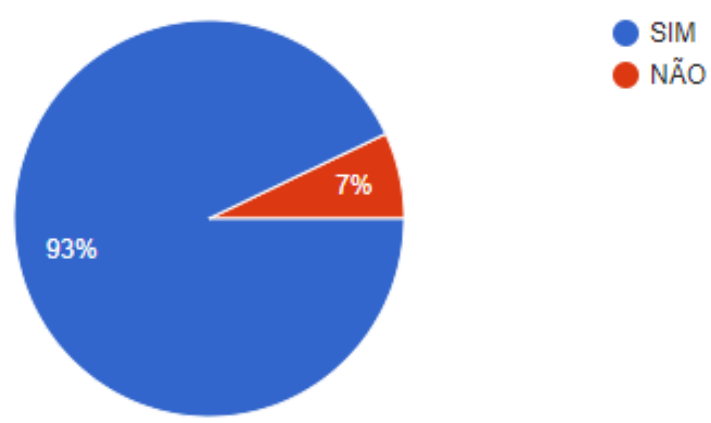

Asimismo, al ítem 04, aquí se observa que el $93 \%$ de las personas investigadas conocen lo que se entiende por libertad de prensa, principal derecho y garantía de la actuación periodística. Entonces, es preciso que los mass media se atengan a los principios de los referidos bienes de actuación.

Ítem 06 - “¿CONSIDERA ABUSIVA LA FORMA COMO LOS MEDIOS DE COMUNICACIÓN REPRESENTAN A LA POBLACIÓN PRESA?"

De las 57 (cincuenta y siete) respuestas registradas, el 42,1\% fueron registradas con SÍ y 57,9\% registradas con NO.

\section{Figura 7}

Resultado del ítem 06
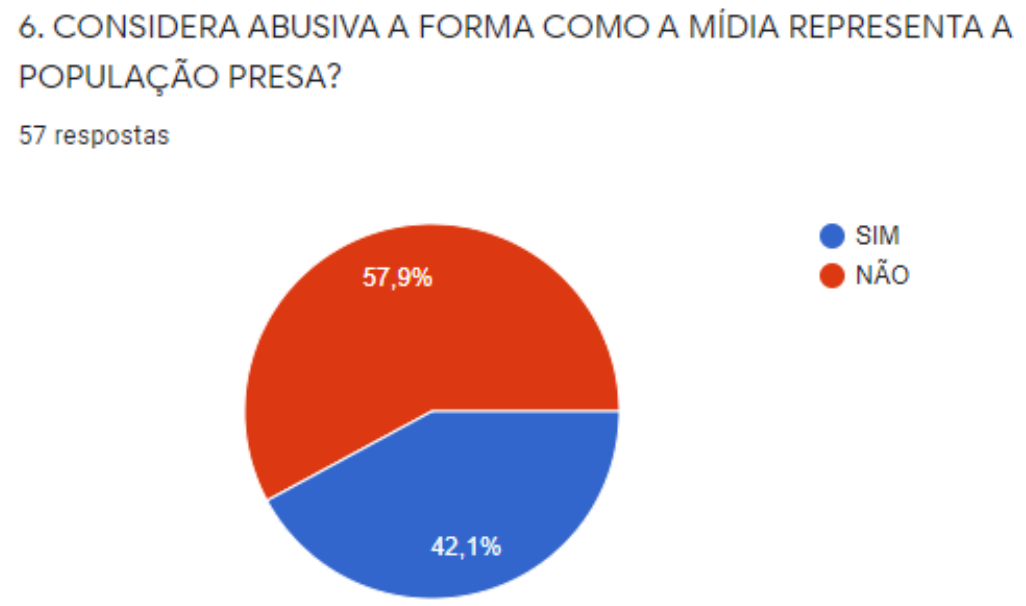
A continuación, se observa que la mayoría de las personas investigadas no consideran que los medios de comunicación reportan a la persona presa de forma abusiva, lo que, en la visión de los investigados, puede atestar, aunque no con precisión, que los derechos de la persona presa y la actuación del Jornal de Tocantins cubriendo crisis en el sistema de prisiones no se muestran irrespetuosos y/o abusivos

A pesar de todo, no comparten la idéntica opinión la otra parte de los investigados en esta sección, que presentan $42,1 \%$, con una diferencia de $15,8 \%$ del punto da la opinión mayoritaria.

Ítem 07 - “ ¿CONSIDERA QUE LA LIBERTAD DE PRENSA ES UN DERECHO DE LOS PERIÓDICOS BRASILEÑOS, PUDIENDO ESTOS VEHÍCULOS, TRATAR EN SUS NARRATIVAS SOBRE LA IMAGEN, LA PRIVACIDAD Y LA INTIMIDAD DE LA PERSONA PRESA? Alternativas: SÍ o NO.

De las 57 (cincuenta y siete) respuestas registradas, 38,6\% fueron registradas con SÍ y $61,4 \%$ registradas con NO.

\section{Figura 8}

Resultado del ítem 07

7. CONSIDERA QUE A LIBERDADE DE IMPRENSA É UM DIREITO DOS

JORNAIS BRASILEIROS, PODENDO ESTES VEICULOS DE NOTICIAS, EM

NOME DA LIBERDADE DE IMPRENSA, TRATAR EM SUAS NARRATIVAS DA

IMAGEM, DA PRIVACIDADE E DA INTIMIDADE DA PESSOA PRESA?

57 respostas

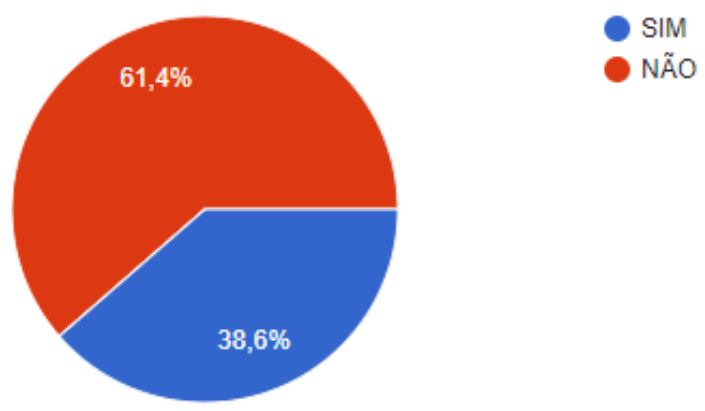

El último ítem reafirma los objetos trabajados en los cuestionamientos pues, en conclusión, pone en línea de embate la actuación periodística (garantizada por la libertad de prensa) y los derechos de las personas presas, precisamente los derechos personalísimos de imagen, privacidad e intimidad.

En este momento, se observa que la mayoría de las personas consideran que los mass media no pueden hacer uso de la imagen, de la privacidad y de la intimidad de las personas presas. Referido dato confirma el grado de consciencia del mayor número de investigados en relación a algunos límites de la actuación periodística en el ambiente de la prisión. 


\section{CONCLUSIONES}

El curso del camino discursivo adoptado en este trabajo ha buscado tratar sobre un delicado escenario, el ambiente carcelario en Tocantins y la actuación de los medios tocantinenses, especialmente el Jornal de Tocantins, para confirmar los efectos de la Teoría de la Planificación o Agenda Setting en la formación de la opinión pública o de la agenda pública de las personas investigadas, profesionales del Sistema de Prisiones, del Periodismo, de la Orden de Abogados de Brasil - OAB, de la Defensoría Pública de Tocantins y de la Procuraduría Federal de Justicia de Tocantins.

El estudio buscó iniciar una discusión en el ámbito de las Teorías de la Comunicación, especialmente, sobre la Agenda Setting entrelazada con el Derecho, a fin de observar como los mass media actúan en el ambiente carcelario en consideración con los derechos de los presos, el ambiente de la delicada realidad que refleja la brutal condición humana ampliamente debatida y que, para más allá de condiciones mínimas de vidas se ve, muchas veces, siendo invadido y violado de cualquier forma y modo por el mundo externo a los muros de la prisión.

Aquí se discutió de forma breve la actuación periodística en la formación de narrativas que han de desaguar en la formación de la agenda pública de la sociedad, lo que puede contribuir, o no, a lo sustento e la dura realidad humana y de los derechos en la cárcel, situación ampliamente debatida y conocida en el medio social, político, académico y científico del Periodismo y del Derecho.

Las conclusiones alcanzadas con la fuerza de los datos empíricos, aunque traten en suficiencia la materia o el tema, requieren la contribución de nuevos estudios, posibilitando entender y comprender de forma más ampliada los efectos de las teorizaciones de la Agenda Setting en el ambiente carcelario y en la formación de la opinión pública.

Como conclusión, los datos presentados y discutidos evidenciaron que la sociedad investigada se muestra consciente de lo que se entiende por libertad de prensa y derechos personales, lo que requiere una actuación periodística más atenta a las elecciones de valores-noticia por parte del Jornal de Tocantins en relación con la formación de la agenda mediática y pública, considerando los estudios de la Planificación, sin ignorar otros sectores sociales, como por ejemplo el Derecho en lo que toca a los derechos personales sobrepujados al ambiente carcelario y a la persona del preso.

Además, el estudio propone nuevas líneas de investigación (bibliográficas o empíricas), con diferentes perspectivas, y capaces de difundir e innovar los estudios de ajuste de la agenda en prisión, que involucran principalmente el desempeño de los periódicos y la población penitenciaria, ya que los datos presentados y discutidos en este estudio demuestran que es necesario conocer, además de lo que ya se ha reflejado, otras posibles influencias de los medios de comunicación en las percepciones de los individuos sobre la población carcelaria, no solo con una mirada centrada en el objeto comunicacional sino también en los objetos que rodean figura del preso: la ejecución de la sentencia, la eficiencia de las políticas penitenciarias, el espacio de segregación y su realidad física, simbólica y humana para comportarse los seres humanos, etc.

Los resultados alcanzados $(47,4 \%$ de las personas afirman considerar confiables las noticias de Jornal do Tocantins; $71,9 \%$ de las personas no se consideran influenciadas por los medios de comunicación; 64,9 \% de las personas afirman haber leído noticias sobre las crisis en el sistema carcelario publicadas por el Jornal do Tocantins; el 71,9 \% de las personas reconoce los derechos del preso a la imagen, la privacidad y la intimidad; 
el $93 \%$ de las personas dice que sabe lo que tiene para la libertad de prensa; el 57,9\% de las personas no considera representación de la persona encarcelada por los medios de comunicación; el 61,4\% de las personas no confirman que la libertad de prensa vincula el uso o la difusión de la imagen, la privacidad y la intimidad de los presos en las noticias) impulsan los estudios de McCombs y Shaw escenario de prisión para la búsqueda de conocer y comprender las verdaderas influencias de los medios de comunicación.

Por lo tanto, los datos antes mencionados confirmaron la influencia de las noticias del Jornal do Tocantins en la opinión pública de quienes consumen el contenido de los medios de ese vehículo, señalando a partir de la muestra presentada, que la programación es por la causa y el momento del efecto sentido lo que da como resultado opiniones que se mostrarán como producto de las influencias recibidas y las percepciones construidas a partir de ellas.

\section{REFERENCIAS BIBLIOGRÁFICAS}

Araújo, C. A. (2008). A pesquisa norte-americana. En A. Hohlfeldt, L.C. Martino, y V.V. França, Teorias da Comunicação (pp. 119-130). Petrópolis: Vozes.

Alonso, L. S. (2016). O corpus documental em história oral: teoria, experiência e transcriação. Revista Observatório, 2(1),

54-75. https://doi.org/10.20873/uft.2447-4266.2016v2n1p54.

Almeida, J. R. (2016). História e oralidade nos estudos comunicacionais. Revista Observatório, 2(2), 47-58. $\quad$ https://doi.org/10.20873/uft.24474266.2016v2especial1p47.

Assembleia Legislativa do Tocantins. (2017). Diário Oficial, Ano XXVI, $n^{\circ} 2427$ (pp. 34). Disponible en: http://al.to.leg.br/diario.

Barros Filho, C. (1995). Ética na comunicação: da informação ao receptor. São Paulo: Summus.

Bitencourt, C. R. (2016). Tratado de Direito Penal: parte geral. 12a ed. São Paulo: Saraiva.

Demo, P. (1994). Pesquisa e construção de conhecimento: metodologia científica no caminho de Habermas. Rio de Janeiro: Tempo Brasileiro.

Ferreira, G. (2000). Aplicação da Pena. Rio de Janeiro: Forense.

Formiga, F. O. N. (2006). A Evolução da Hipótese de Agenda-setting. Disponible en: http://livros01.livrosgratis.com.br/cp049726.pdf.

Foucault, M. (1975). Vigiar e punir: nascimento das prisões. Trad. Ramalhete, R. Petrópolis: Vozes.

Gerhardt, T. E. y Silveira, D. T. (2009). Métodos de pesquisa. Porto Alegre: Editora da UFRGS.

Gil, A. C. (1991). Como elaborar projetos de pesquisa. São Paulo: Atlas.

Lippman, W. (2008). Opinião pública. Petrópolis: Vozes.

Luo, Y. (2011). The internet and politics in China: the agenda-setting influence of online public opinion on media coverage and government policy. Disponible en: https://ijoc.org/index.php/ijoc/article/view/2257/1133.

Martinez, M. (2016). Reflexões sobre Jornalismo e História Oral: um campo com mais convergências do que dissonâncias. Revista Observatório, 2(1), 75-91. https://doi.org/10.20873/uft.2447-4266.2016v2n1p76.

Martino, L. M. S. (2010). Teoria da Comunicação: ideias, conceitos e métodos. Petrópolis: Editora Vozes. 
Mccombs, M. (2004). A teoria da agenda: a mídia e a opinião pública. Petrópolis: Vozes. Mccombs, M., y Shaw, D. (1972). The agenda-setting function of mass media (pp. 176182). Public Opinion Quaterly.

Miotto, A. B. (2020). O direito penitenciário: importância e necessidade do seu estudo. Disponible en: http://www2.senado.leg.br .

Mirabete, J. F. (1996). Execução Penal: comentários à Lei, n. 7.210. São Paulo: Atlas.

Melo, A. S. E. (2017). Enquadramento histórico legal do processo de Bolonha e o seu impacto no sistema de ensino superior português. Revista Observatório, 3(6), 75141. https://doi.org/10.20873/uft.2447-4266.2017v3n6p75.

Moio, I., A., L. y Vieira, C. C. (2017). A declaração de Bolonha e o reforço da abertura do ensino superior a novos públicos: perceções de pessoas adultas que frequentam a Universidade de Coimbra. Revista Observatório, 3(6), 169-201. https://doi.org/10.20873/uft.2447-4266.2017v3n6p169.

Nunes, S. G., Porto Junior, F. G. R. y Moraes, N. R. de. (2017). Conhecimento e organização: indicativos pós-Bolonha de uma sociedade em construção. Revista Observatório, 3(6), 338-353. $\quad$ https://doi.org/10.20873/uft.24474266.2017v3n6p338.

Oliveira, E. (1997). Politica criminal e alternativas à prisão. Rio de Janeiro: Forense.

Porto Junior, F. G. R. y Moraes, N. R. de. (2017). Formando pesquisadores pós-bolonha em portugal: relações entre a formação de graduação e o campo da pesquisa/investigação. Revista Observatório, 3(6), 202-228. https://doi.org/10.20873/uft.2447-4266.2017v3n6p202.

Reis, E. G. dos. (2017). O ensino superior em cabo verde e os desafios do processo de Bolonha. Revista Observatório, 3(6), https://doi.org/10.20873/uft.2447-4266.2017v3n6p142.

Rovai, M. G. de O. (2017). HISTÓRIA PÚBLICA: a comunicação e a educação histórica. Revista Observatório, 3(2), 41-65. https://doi.org/10.20873/uft.24474266.2017v3n2p41.

Secretaria de Cidadania e Justiça do Tocantins. (2018). Relatório de Gestão. Disponible en: https://central3.to.gov.br/arquivo/406567/ .

Secretaria de Cidadania e Justiça do Tocantins. (2018). Relatório com controle da População Carcerária. Disponible en: https://cidadaniaejustica.to.gov.br/institucional/institucional/relatorio-de-gestaol.

Secretaria de Cidadania e Justiça do Tocantins. (2017). Plano de Adequação do Sistema Penitenciário do Tocantins. Disponible https://www.cge.to.gov.br/relatorios/prestacao-de-contas/relatorios-deprestacaode-contas-2017/secretaria-da-cidadania-e-justica---seciju-2017/secretaria-dacidadania-e-justica---seciju-2017/.

Wolf, M. (2002). Teorias da Comunicação. Lisboa: Editora Presença. 